\title{
Staatliche Systeme sozialer Sicherheit in Afrika: Das Beispiel staatlicher Alterssicherung in Ghana 1
}

\author{
Von Ulrike Wanitzek
}

\section{Einleitung}

Etwa 5,8\% der Weltbevölkerung sind älter als 65 Jahre. Obwohl der Anteil der alten Bevölkerung in den Industrieländern mit $11,3 \%$ wesentlich höher liegt als in den Entwicklungsländem mit $4 \%$ (Afrika: $3 \%$ ), ist die absolute Zahl der über 65 jährigen in den Ländem der sogenannten Dritten Welt mit 132 Millionen um 4 Millionen höher als in den Industrieländern; und aufgrund stärker steigender Geburtenraten wird angenommen, daß um das Jahr 2020 die alte Bevölkerung in der Dritten Welt in absoluten Zahlen mehr als doppelt so stark sein wird als die in den Industrieländern.2

Während in den Industrieländern die Regelung der Altersversorgung heute weitgehend als Aufgabe des Staates angesehen wird, gelten in vielen Ländern der Dritten Welt ${ }^{3}$ einschließlich der afrikanischen Länder staatliche Maßnahmen der Altersversorgung nach wie vor als lediglich ergänzende Maßnahmen, die neben die Sorge der Familien und sonstigen Gemeinschaften für ihre alten Mitglieder treten. ${ }^{4}$ In den traditionellen afrikanischen Gesellschaften bedeutete Alter die höchste Stufe von Autorität, Macht, Privilegien, und

1 Die Autorin dankt Herm Prof. W.C. Ekow Daniels für seine unschätzbare Hilfe bei der Materialbeschaffung für diesen Aufsatz sowie für zahlreiche Erläuterungen hierzu; und Dr. Eva-Maria Hohnerlein für die kritische Durchsicht des Manuskripts.

2 Laslett, Peter: The Demographic Scene - An Overview. In: Eekelaar, John M./Pearl, David (eds.), An Aging World. Dilemmas and Challenges for Law and Social Policy, Oxford, Clarendon Press, 1989, S. 3-19, auf S. 4 f.

3 Zum Begriff der "Dritten Welt" im Zusammenhang mit länderübergreifenden Aussagen auf dem Gebiet der sozialen Sicherheit vgl. Fuchs, Maximilian: Soziale Sicherheit in der Dritten Welt. Zugleich eine Fallstudie Kenia, Baden-Baden, Nomos Verlagsgesellschaft, 1985, S. 13.

4 Zacher, Hans F.: Traditional Solidarity and Modem Social Security: Harmony or Conflict? In: von Benda-Beckmann, Franz, et alii (eds.), Between Kinship and the State: Social Security and Law in Developing Countries, Dordrecht, Foris Publications, 1988, S. 21-38, auf S. 32; Rwezaura, Barthazar A.: Changing Community Obligations to the Elderly in Contemporary Africa. In: Eekelaar, John M./Pearl, David (eds.), An Aging World. Dilemmas and Challenges for Law and Social Policy, Oxford, Clarendon Press, 1989, S. 113-131, auf S. 126 f. 
Prestige. ${ }^{5}$ Alte Menschen waren aufgrund ihrer hiernit verbundenen Verfügungsgewalt über wirtschaftliche Ressourcen, wie insbesondere Boden und Vieh, relativ abgesichert. 6 Jedoch ist trotz der vielfach anzutreffenden Auffassung, daß die "extended family" in Afrika auch heute noch funktioniere und die Versorgung ihrer schwächeren Mitglieder sicherstelle, nicht zu übersehen, daß sich sowohl die Möglichkeiten als auch die Bereitschaft der erweiterten Familie, für ihre schwächeren Mitglieder zu sorgen, verringert haben. 7 Denn mit den gesellschaftlichen und wirtschaftlichen Veränderungen während und nach der Kolonialzeit verloren traditionelle Formen sozialer Sicherheit in Afrika zunehmend an Effektivität und Verläßlichkeit. Die Folgen von Verstädterung, Industrialisierung, Migration, Lohnarbeit, und allgemein der Einführung der Geldwirtschaft ließen Lücken im sozialen Netz entstehen, 8 die auch die Altersversorgung betreffen: "Old age became a disability as well as an economic risk".9

Auch in Ghana ist eine Krise der traditionellen Formen sozialer Sicherheit, einschließlich der Alterssicherung, zu beobachten. 10 Es stellt sich daher die Frage, inwieweit der Staat mit seinen Maßnahmen zur Alterssicherung in der Lage gewesen ist, diese Lücken im sozialen Netz zumindest teilweise zu schließen.11 Bereits während der Kolonialzeit wurden Pensionsgesetze erlassen, die eine Altersversorgung der in der Kolonialverwaltung tätigen

5 Apt, Nana A.: Urbanization and the Aged. In: Oppong, Christine (ed.), Legon Family Research Paper No. 3: Changing Family Studies, Legon, Institute of African Studies, 1975, S. 177-183, auf S. 177.

6 Rwezaura, op. cit., S. 113; Omari, C.K.: The Future of the Elderly in the African Family. Paper, Center for International and Comparative Studies, University of Iowa, USA, 1987, S. 6.

7 Rwezaura, op. cit., S. 127.

8 Apt, op. cit., S. 177 ff.; Asamoah, Yvonne/Nortey, DN.A.: Ghana, In: Dixon, John (ed.), Social Welfare in Africa, London etc., Croom helm, 1987, S. 22-68, auf S. 34; Fuchs, op. cit., S. 38 f.; Tumer, E.: Report on the Setting up of a Contributory Social Security Scheme with Particular Reference to the Needs of Persons in their Old Age, Entebbe, Government Printer, 1964, S. $4 \mathrm{ff}$. Zur internationalen Migration vgl. Kaufmann, Otto: Internationale Aspekte der sozialen Sicherheit in den francophonen Ländern südlich der Sahara, Verfassung und Recht in Übersee, 18 (1985), S. 25-36, auf S. 26.

9 Rwezaura, op. cit., S. 113.

10 Apt, op. cit., S. 177 f.; Schott, Rüdiger: Traditional Systems of Social Security and their PresentDay Crisis in West Africa. In: von Benda-Beckmann, Franz, et alii (eds.), Between Kinship and the State: Social Security and Law in Developing Countries, Dordrecht, Foris Publications, 1988, S. 89-107, auf S. 101 ff.; Kludze, AK.P.: Formal and Informal Social et alii (eds.), Between Kinship and the State: Social Security and Law in Developing Countries, Dordrecht, Foris Publications, 1988, S. 187-209, auf S. 208.

11 Zur Situation staatlicher Alterssicherung in Tansania vgl. Bossert, Albrecht: Traditionelle und moderne Formen sozialer Sicherheit in Tanzania. Eine Untersuchung ihrer Entwicklungsbedingungen, Berlin, Duncker \& Humblot, 1985; Wanitzek, Ulrike: State Provisions for Old-Age Security in Tanzania. In: Eeekelaar, John M./Pearl, David (eds.), An Aging World. Dilemmas and Challenges for Law and Social Policy, Oxford, Clarendon Press, 1989, S. 899-921; zu Kenia vgl. Fuchs, op. cit. 
europäischen und afrikanischen Beamten vorsahen. Nach der Unabhängigkeit folgten Gesetze, die auch die Altersversorgung sonstiger staatlicher Bediensteter sowie privater Arbeitnehmer regelten. Da diese Gesetze jedoch das Problem der Altersversorgung nicht in befriedigendem Maße lösen konnten, wurde 1988 ein Entwurf eines grundlegend neuen Rentensystems ausgearbeitet, das "Social Security and National Insurance Trust (SSNIT) Pension Scheme". Es wird erwartet, daß der Provisional National Defence Council (PNDC) 12 demnächst auf der Grundlage dieses Entwurfs ein neues Rentengesetz erlassen wird.13 In dem vorliegenden Beitrag wird zunächst ein Ưberblick über die Ausgestaltung der beiden bisher geltenden Systeme staatlicher Alterssicherung - Public Pension Scheme und Social Security Fund - gegeben, 14 und dann der Entwurf des neuen Rentengesetzes das SSNIT Pension Scheme - vorgestellt. Dabei werden Probleme und Unzulänglichkeiten diskutiert, die sich bei der Anwendung der bestehenden Systeme ergeben und die einerseits zu der Erarbeitung eines neuen Rentengesetzes führten, andererseits durch dieses sicher nicht immer gelöst werden können.

\section{Das Pensionssystem für Beamte (Public Pension Scheme)}

\section{Allgemeines}

Das Public Pension Scheme, das Pensionssystem für Beamte,15 ist das älteste System staatlicher Alterssicherung in Ghana. Die ersten kolonialen Pensionssysteme hatten noch

12 Der ghanaische Militärrat, der sowohl Exekutiv- als auch Legislativfunkion wahmimmt.

13 Vgl. die Berichte in der ghanaischen Presse: The Mirror, Tageszeitung, Accra, 19.03.88 und 07.07.90; People's Daily Graphic, Tageszeitung, Accra, 04.02.88 und 02.06.90. In Intemational Social Security Association (ISSA): XXIIIrd General Assembly, Vienna, 5-13 September 1989. Developments and Trends in Social Security 1987-1989. Report of the Secretary General, Part I. ISSA/GA/XXIII/1, 1989, S. 11, wurde fälschlicherweise berichtet, daß der Rentenrechtsentwurf bereits Gesetz sei. Dies war jedenfalls bis zum August 1990 noch nicht der Fall.

14 Aufgrund der zahlreichen Regierungswechsel in Ghana haben sich die Gesetzesbezeichnungen häufig geändert. Die folgenden gängigen Abkürzungen für Gesetzesbezeichnungen erscheinen im folgenden Text: Cap. (Chapter, Laws of the Fold Coast, Rev. Ed. 1951 ff.); C.A. (constituent Assembly Act); NLCD (National Liberation Council Decree); NRCD (National Redemption Council Decree); SMCD (Supreme Military Council Decree); PNDCL (Provisional National Defence Council Law). Nebengesetzgebung: L.I. (Legislative Instrument). Des weiteren stehen s. für section, art. für article, und r. für regulation. Ein vollständiger Überblick über die unter den verschiedenen Regierungen verwendeten Gesetzesbezeichnungen findet sich bei Daniels, Janet: Thana. In: Intemational Encyclopedia of Comparative Law, vol. I, National Reports, Tübingen etc., 1972, G33-47.

15 Näheres zum Beamtenbegriff s.u. 
zwischen Europäem und Afrikanem differenziert. ${ }^{16}$ Bis in die Zeit nach dem 2 . Weltkrieg wurden zumeist nur die niederen Beamtenposten mit Afrikanem besetzt, während die höheren Stellen Europäem vorbehalten waren.17 Dies erklärt sich mit dem Selbstverständnis und den Funktionen der Kolonialverwaltung, die in erster Linie die imperiale Politik Großbritanniens in seinen abhängigen Territorien durchzusetzen hatte; eine interne Selbstverwaltung der Afrikaner oder gar Unabhängigkeit dieser Territorien wurde bis in die Jahre nach dem 2. Weltkrieg für mindestens eine weitere Generation für ausgeschlossen gehalten. 18 Soweit höhere Posten ausnahmsweise doch mit Afrikanem besetzt wurden, geschah dies einerseits zur Befriedigung der Forderungen der wenigen gebildeten Afrikaner und einflußreicher Chiefs, sowie um andererseits den Forderungen der liberalen englischen Parlamentsangehörigen nachzukommen.19 Erst 1946 wurde, in Folge der Arbeit der Harragin Salaries Structure Commission, diese auf Rassenbasis vorgenommene Aufgliederung der Beamtenschaft in einen afrikanischen "junior service" und einen europäischen "senior service" abgeschafft.20 Mit Wirkung vom 01.01.1946 galt auch die - nicht mehr zwischen Europäern und Nicht-Europäem differenzierende - Pensions Ordinance von 1950 (Cap. 30).

Die Struktur des kolonialen öffentlichen Dienstes wurde vom unabhängigen Staat Ghana aus Praktikabilitätsgründen übernommen.21 Der öffentliche Dienst (public services) umfaßt die in der allgemeinen Staatsverwaltung tätigen Beamten (civil service), sowie die sonstigen öffentlichen Bediensteten im Justizdienst (judicial service), im Rechnungsdienst (audit service), im Schuldienst (education service), im Strafvollzug (prisons service), im Parlamentsdienst (Parliamentary service), im Gesundheitswesen (health service), im statistischen Dienst (statistical service), bei der Feuerwehr (fire service), im Zolldienst (customs and preventive service), in bestimmten öffentlich-rechtlichen Körperschaften (public corporations service), sowie in sonstigen durch Verfassung oder Gesetz errichteten Diensten.22

16 Vgl. z.B. Pensions (Non-European Officers) Ordinance, Cap. 126; Pensions (Non-European Officers War Service) Ordinance, 1940; Pensions (European Officers) (Old Conditions of Service Pensions) Ordinance, 1940.

17 In Ostaf rika gab es außerdem noch eine mittlere Beamtenschicht, die sich aus den Mitgliedem der eingewanderten europäischen und asiatischen Gemeinschaften rekrutierte.

18 Adu, AL.: The Civil Service in Commonwealth Africa. Development and Transition, London, George Allen \& Unwin, 1969, S. 19.

$19 A d u$, op. cit., S. 20.

20 In Ostafrika geschah dies erst 1954, aufgrund der Empfehlungen der Lidbury Commission, Adu, op. cit., S. 21.

21 Adu, op. cit., S. $32 \mathrm{ff}$.

22 Art. 154(1) Constitution 1979, bestätigt durch s. 11 PNDC (Establishment) Proclamation (1981) und s. 37 PNDCL 42 (1982). Die "public services" werden definiert als "service in any civil capacity of the Govemment, the emoluments attached to which are paid directly from the consolidated Fund of directly out of moneys provided by Parliament and the service with any statutory corporation established entirely out of public funds or moneys provided by Parliament"; und die Angehö- 
Der in dieser Auflistung als erstes genannte "civil service", der die in der allgemeinen Staatsverwaltung tätigen Beamten umfaßt, wird definiert als alle Staatsdiener (ausgenommen die jenigen, die ein politisches Amt innehaben oder im Justizdienst tätig sind), die in ziviler Eigenschaft angestellt sind, und deren Gehälter vollständig und direkt aus vom Parlament 23 bewilligten Haushaltsgeldem finanziert werden.24 Die Ausgestaltung des "civil service" findet sich im Civil Service Act von 1960 (C.A. 5), und die Pensionsregelungen für Beamte in der Pensions Ordinance von 1950 (Cap. 30). Die Sonderstellung der Beamten beruht darauf, daß sie die Kerngruppe des öffentlichen Dienstes darstellen, der die Durchführung der politischen Entscheidungen der Regierung anvertraut ist.25

Die anderen, oben aufgezählten Berufsgruppen des öffentlichen Dienstes, sowie der Polizei- und der Militärdienst, haben aufgrund zahlreicher Besonderheiten ihre eigenen gesetzlichen Ausgestaltungen erfahren.26 Dies gilt auch für die Regelungen zur Altersversorgung.27 Diese sollen jedoch in ihren Details hier nicht näher untersucht werden; im folgenden werden vielmehr die Grundzüge des allgemeinen ghanaischen Pensionssystems für Beamte dargestellt.

\section{Pensionsberechtigung}

Nicht alle Angehörigen des "civil service" sind pensionsberechtigt. Die gesetzlichen Bestimmungen darüber, wann jemand ein "pensionable office" innehat, differenzieren insbesondere nach Art und Dauer der Tätigkeit eines Beamten. 28 Soweit bestimmte Beamtengruppen nicht pensionsberechtigt sind, wird ihre Altersversorgung im Rahmen anderer Systeme, wie des Social Security Fund (s.u.) und gewerkschaftlich ausgehandelter Systeme, 29 geregelt. 30

rigen des öffentlichen Dienstes, die "public officers", sind "those persons who actually carry out executive directives under the authority of Commissioners of State", Daniels, W.C. Ekow: The Meaning and Scope of Executive Power in Ghana today. University of Ghana Law Joumal, 11(1974), S. 109-121, auf S. 120.

23 Die Funktionen des Parlaments werden heute vom PNDC wahrgenommen.

24 Adu, op. cit., S. 26.

25 Daniels, op. cit., S. 121.

26 Vgl. dazu Adu, op. cit., S. 212 ff; Daniels, op. cit., S. 120 f.

27 Z.B. Teachers Pensions Ordinance, 1955 (23/55); Armed Forces Act, 1962 (Act 105); Armed Forces Pensions (Civilian Employees) Decree, 1967 (NLCD 166) i.V.m. Amd., 1977 (SMCD 106); Police Service Act, 1970 (Act 359); Police Service Pensions Law, 1985 (PNDCL 126).

28 Vgl. Civil Service Act, 1960 (C.A. 5).

29 Die Civil Service Association ist z.B. eine gewerkschaftliche Beamtenvereinigung.

30 Adu, op. cit., S. 33 f. 
Das Public Pension Scheme ist beitragsfrei, d.h. die Beamten haben keine eigenen Beiträge zu erbringen. Die Pensionsleistugen werden vielmehr durch das allgemeine Steueraufkommen des Staates finanziert. 31

\section{Leistungsvoraussetzungen und Leistungsinhalt}

Das freiwillige Pensionsalter liegt bei 45 Jahren,32 und das obligatorische Pensionsalter bei 60 Jahren. 33

Einer Beamtin, die wegen ihrer bevorstehenden Heirat oder während einer bestehenden, während ihrer Dienstzeit geschlossenen Ehe in den Ruhestand tritt, aber die Alters- oder sonstigen Anforderungen für einen Pensionsanspruch noch nicht erfüllt, kann eine Sonderleistung gewährt werden.34 Andererseits kann eine Beamtin, die heiratet oder verheiratet ist, aus diesem Grund von ihrem Dienstherm jederzeit aufgefordert werden, in den Ruhestand zu treten. 35

Mitglieder des Pension Scheme haben kein uneingeschränktes Recht auf die Pensionsleistungen. Sie können jederzeit, unter Verlust ihrer Pensionsanwartschaft, entlassen werden und können nicht gegen den Staat auf Zahlung ihrer Pension klagen.36 Auch aufgrund strafrechtlicher Vergehen, die nicht unbedingt in Zusammenhang mit den Anforderungen seines Amtes stehen müssen, kann ein Beamter disqualifiziert werden und damit seine Pensionsansprüche ganz oder teilweise verlieren. 37

Pensionsansprüche sind nicht übertragbar, außer um eine Schuld an die Regierung zu begleichen oder um ein gerichtliches Unterhaltsurteil für Ehefrau oder minderjährige Kinder zu erfüllen.38 Bei Konkurs des Pensionsverpflichteten enden die Pensionslei-

31 S. 5 Pensions ord.

32 S. 7 (1) Pensions Ord.; art. 162(2) Constitution 1979, bestätigt durch s. 2 PNDCL 23. Weitere Entstehungsgründe, neben Alter, für einen Pensionsanspruch: s. 7(2) ff. Pensions Ord.

33 Art. 162(1) Constitution 1979; s. 1 PNDCL 23. Sonderregelungen hinsichtlich des Pensionsalters bestehen, wie oben bereits erwähnt, z.B. für Richter, Militär-, Polizei- und Universitätsangehörige, vgl. die einzelnen Spezialgesetze und s. 4 PNDCL 23.

34 S. 7 Pensions Ord., 1. proviso; vgl. auch rr. 6,13 Pensions Reg.

35 S. 8(2), (3) Pensions Ord. Das gleiche gilt für Beamte, die über 45 Jahre alt und im Amt geblieben sind.

36 Daniels, op. cit., S. 122.

37 S. 6 Pensions Ord.

38 S. 12 Pensions Ord. 
stungen, ${ }^{39}$ ebenso bei Verurteilung zu einer Freiheitsstrafe 40 und bei Emennungen in bestimmte Ämter. 41

Die Pension wird monatlich ausgezahlt. Der Beamte hat die Möglichkeit, monatliche Leistungen lediglich in Höhe von drei Vierteln der vollen Pension mit der Zahlung einer einmaligen Pauschalsumme zu kombinieren, die den zwanzigfachen Betrag der Differenz zwischen voller und reduzierter Pension beträgt.42 Diese Kombination ist beliebt und wird vielfach praktiziert, weil der Pensionierte hierdurch sofort einen gewissen Geldbetrag in Händen hat. Die Pensionsbezüge sind steuerfrei.43 Die Pension darf das pensionsfähige Einkommen des Beamten, das er unmittelbar vor seiner Pensionierung hatte, nicht übersteigen.44 Der Pensionsfaktor, der für die Höhe der Leistungen relevant ist, wurde 1975 von $1 / 60045$ auf 1/48046 heraufgesetzt, d.h. die Pension beträgt 1/480 des jährlichen pensionsfähigen Einkommens pro Monat des Dienstverhältnisses. 47 Bei emeutem Eintritt des pensionierten Beamten in den öffentlichen Dienst können seine Pensionsbezüge solange ausgesetzt werden, wenn dies angemessen erscheint und er zustimmt.48

Im Falle eines Arbeitsunfalls kann eine zusätzliche Pension gewährt werden.49 Stirbt ein Beamter während seiner Dienstzeit, so steht seinem Erbschaftsverwalter sowie einem von dem Beamten vor seinem Tode benannten Verwandten eine Gratifikation bis zum Betrag seines pensionsfähigen Jahreseinkommens zu.50 Dem Ehepartner51 des verstorbenen Beamten kann eine Pension bis zur Höhe von einem Viertel des pensionsfähigen Einkommens des Beamten vor seinem Tode gewährt werden, solange er unverheiratet bleibt. 52 Seinen Kindem kann, bis sie das 21. Lebensjahr erreichen und solange sie unverheiratet bleiben, eine Pension bis zu 1/16 bzw. 1/8 des pensionsfähigen Einkommens des Beamten

39 S. 13 Pensions Ord.

40 S. 14 Pensions Ord.

41 S. 15 Pensions Ord.

42 R. 26(1) Pensions Reg., in der Fassung von NRCD 74.

43 Art. 162(3) Constitution 1979; s. 3 PNDCL 23.

44 S. 10 Pensions Ord., in der Fassung von s. 5 NRCD 74 i.V.m. s. 1 SMCD 166 . Vorher lag die Höchstpension bei zwei Dritteln des höchsten pensionsfähigen Einkommens, das der Beamte im Laufe seiner Beschäftigung hatte (s. 10(2) Pensions Ord. a.F.).

45 R. 4(b) Pensions Reg. a.F.

46 R. 4(b) Pensions Reg., in der Fassung von s. 12(1) SMCD 8.

47 Die Pensionsformel lautet: Jährliche Pension = Pensionsfaktor $\mathrm{x}$ pensionsfähiges Jahreseinkommen $\times$ Zahl der Gesamtmonate des Dienstverhältnisses.

48 S. 11 Pensions Ord.

49 R. 24 Pensions Reg.

50 S. 16 Pensions Ord.

51 Ursprünglich: die Witwe, vgl. dazu auch Fuchs, op. cit., S. 29; seit 1975 geschlechtsneutral formuliert, SMCD 8.

52 S. 17(1) (i) Pensions Ord. 
vor seinem Tod gewährt werden. ${ }^{53}$ Auch nichteheliche Kinder, Stiefkinder und Adoptivkinder, die von dem Verstorbenen wirtschaftlich abhängig waren, fallen unter den Begriff "Kind".54

Eine Sondervorschrift für polygame Ehen bestimmt, daß das für den Ehepartner bestimmte Viertel, sowie die für die Kinder bestimmten Anteile, auf mehrere Witwen bzw. auf die Kinder aus mehreren Ehen des Verstorbenen aufgeteilt werden können, 55 was allerdings zu extrem niedrigen Beträgen pro Person führt.56 Schließlich können auch die Eltern des Verstorbenen, wenn sie wirtschaftlich von ihm abhängig waren und solange sie dies bleiben, eine Pension in der gleichen Höhe wie der Ehepartner, d.h. bis zu 1/4, erhalten; Voraussetzung ist jedoch, daß kein Ehepartner hinterblieben ist oder ihm keine Pension gewährt wurde. 57

\section{Probleme des Public Pension Scheme}

Das Hauptproblem des Public Pension Scheme liegt darin, daß kein uneingeschränkter Rechtsanspruch auf die Pension besteht und ein Beamter jederzeit unter Verlust jeglicher Altersversorgung entlassen werden kann. Des weiteren sind die Leistungen gewöhnlich viel zu niedrig, um davon leben zu können. Bereits die vollen Gehälter reichen nicht aus, um auch nur die bescheidensten Lebenshaltungskosten decken zu können. Dieses Problem entstand aufgrund der ständig wachsenden Inflations- und Abwertungsrate und verschärft sich weiter. 58

Vorteile des Pension Scheme liegen darin, da $\beta$ die Pensionsleistungen steuerfrei sind; 59 da $\beta$ es sich im Gegensatz zu dem Social Security Scheme (s.u.) um ein echtes Sozialversicherungssystem und nicht um ein bloßes Zwangssparsystem handelt, und daß seit einigen Jahren die Auszahlung der Altersleistungen wohl relativ zuverlässig und unproblematisch funktioniert.60

53 Dies ist auf sechs Kinder beschränkt, s. 17(1) Pensions Ord., proviso (a). Sind mehr Kinder vorhanden, müssen sie sich - in der Praxis - die für sechs Kinder gewährte Pension teilen.

54 S. 17(4) Pensions Ord.

55 S. 17(1) Pensions Ord., proviso (d).

56 Vgl. auch Fuchs, op. cit. S. 28, zu der Situation bei polygamen Ehen.

57 S. 17(1) Pensions Ord. Zum Verhältnis zwischen Pensionsansprüchen der Hinterbliebenen, Erbrecht und Unterhaltsrecht vgl. In Re Allotey (Decd.); Allotey and Another v. Otoo and Another, Accra H.C. 21.3.80, unveröffentlicht.

58 S. dazu unten.

59 Dies ist z.B. nicht der Fall in Tansania, vgl. Wanitzek, op. cit.

60 Mündliche Auskunft von Prof. W.C. Ekow Daniels, August 1990. Dies ist in anderen Ländem nicht unbedingt der Fall, vgl. z.B. zu Tansania Wanitzek, op. cit. 


\section{Der Social Security Fund}

\section{Allgemeines}

Das im anglophonen Afrika am weitesten verbreitete Alterssicherungssystem, das allerdings auch im Vergleich zu anderen Systemen die geringste Effizienz besitzt, sind die National Provident Funds, d.h. landesweite Vorsorgefonds, die für die soziale Alterssicherung privater Arbeitnehmer zur Verfügung stehen.61 Ein derartiger Fonds mit der Bezeichnung Social Security Fund wurde in Ghana 1965 durch den Social Security Act (Act 279) gegründet und 1972 durch das Social Security Decree (NRCD 127)62 neu geregelt. Der Social Security Fund ist kein System sozialer Sicherheit im engeren Sinn, sondem kann eher als Zwangssparsystem bezeichnet werden.63 Die Leistungen bestehen lediglich in einer Auszahlung der Beiträge, die der betreffende Arbeitnehmer während seiner Erwerbstätigkeit angespart hat, sowie der Beiträge seines Arbeitgebers, zusätzlich der angefallenen Zinsen.64

Von 1965 bis 1972 wurde der Social Security Fund von der State Insurance Corporation verwaltet, und 1972 von dem durch das Social Security Decree gegründeten Social Security and National Insurance Trust (SSNIT) übernommen.65 Der SSNIT wird von einem Verwaltungsrat (Board of Directors) verwaltet. Die primäre Finanzierungsquelle sind die Mitgliederbeiträge sowie die durch deren Anlage erzielten Einkommen. Der Staat leistet keine direkten Finanzierungsbeiträge.

\section{Mitgliedschaftsvoraussetzungen}

Pflichtmitglieder des Social Security Fund sind Arbeitgeber und Arbeitnehmer in Betrieben mit mindestens fünf Angestellten.66 Ausgenommen sind, neben den nach der Pensions Ordinance pensionsberechtigten Beamten, auch die Angehörigen des Militärdienstes und der Universitäten, die ihre eigenen Pensionssysteme besitzen, sowie Angehörige des diplo-

61 Fuchs, op. cit., S. 27.

62 Vgl. auch die Ausführungsbestimmungen hierzu: Social Security Regulations, 1973 (L.I. 818).

63 International Labour Office (ILO): Introduction to Social Security, Geneva, 1984, S. 6.

64 S. 40(2) NRCD 127.

65 S. 2 NRCD 127.

66 S. 23 NRCD 127. In Tansania: mindestens vier Arbeitnehmer, Bossert, op. cit., S. 110; in Kenia und Sambia sind keine Mindestarbeitnehmerzahlen für die Pflichtmitgliedschaft festgelegt, Fuchs, op. cit., S. 163; Mulozi, S.L.: The Role of Social Insurance in Developing Countries. An Example from Africa (Zambia). In: Köhler, PA./Zacher, $H F$. (Hg.), Beiträge zu Geschichte und aktueller Situation der Sozialversicherung. Colloquium des Max-Planck-Instituts für ausländisches und intemationales Sozialrecht, Berlin, Duncker \& Humblot, 1983, S. 579-587, auf S. 580. 
matischen Dienstes. ${ }^{67}$ I.ü. befreit die Existenz eines anderen, z.B. betrieblichen, Provident Fund Arbeitgeber und Arbeitnehmer nicht von dieser Pflichtmitgliedschaft im Social Security Fund.68 Viele Arbeitnehmer haben in der Tat neben dem Social Security Scheme zusätzliche, durch Tarifverträge festgelegte betriebliche Alterssicherungen. Arbeitgeber kleinerer Unternehmen als mit fünf Arbeitnehmern können, mit Zustimmung ihrer Angestellten, einen Antrag auf freiwillige Mitgliedschaft stellen.69 Auch Selbständige, d.h. insbesondere die kleinen, im informellen Sektor tätigen Handwerker, Händler, etc., können auf freiwilliger Basis Mitglieder werden.70 Dies ist allerdings in der Praxis wohl selten der Fall gewesen. Im Gegenteil haben Arbeitgeber versucht, der Sozialversicherungspflicht zu entgehen, indem sie die Zahl ihrer Angestellten dadurch manipulierten, daß sie diese lediglich als Gelegenheitsarbeiter auf Tageslohnbasis einstellten.

Aufgrund NRCD 190 und SMCD 871 konnten die Angehörigen des öffentlichen Dienstes, ausgenommen die Beamten der allgemeinen Staatsverwaltung, 72 innerhalb eines Jahres (1972) zwischen der Mitgliedschaft in ihrem bisherigen Pension Scheme und der Mitgliedschaft im Social Security Scheme wählen.

Die Beiträge werden zu $5 \%$ vom Arbeitnehmer und zu 12,5\% vom Arbeitgeber erbracht. Die Beiträge der Arbeitnehmer werden an der Quelle abgezogen.73 Selbständige haben den Beitragssatz von $12,5 \%$ zu erbringen.74 Werden die Beiträge nicht pünktlich bezahlt, so werden $3 \%$ des unbezahlten Beitrags zusätzlich als Strafzuschlag erhoben.

\section{Leistungsvoraussetzungen und Leistungsinhalt}

Das Social Security Scheme sieht Leistungen bei Alter, Invalidität, Tod, Auswanderung, Krankheit und Arbeitslosigkeit vor.75 Wurden während der Beschäftigungsdauer z.B. Leistungen im Fall von Krankheit bereits in Anspruch genommen, so reduziert dies den für die Altersversorgung zur Verfügung stehenden Betrag.

67 S. 23 NRCD 127 i.V.m. Schedule.

68 S. 24(1) NRCD 127.

69 S. 25(1) NRCD 127.

70 S. 25(5) NRCD 127.

71 Vgl. auch SMCD 178.

72 Zum Polizeidienst vgl. Police Service (Pensions) Law, 1985 (PNDCL 126).

73 S. 27 NRCD 127.

74 S. 27(4) NRCD 127.

75 S. 40 NRCD 127. Die soziale Sicherung im Todesfall oder bei Arbeitslosigkeit ist in ihrer Wirksamkeit allerdings äußerst beschränkt, Kludze, op. cit., S. 190 f. 
Hat ein männlicher Arbeitnehmer das Alter von 50, und eine Arbeitnehmerin das von 45 Jahren erreicht, 76 so kann er/sie in den Ruhestand treten und ist berechtigt, Altersleistungen (superannuation benefits) zu erhalten; wird dies nicht in Anspruch genommen, so können Männer maximal bis zum Alter von 55 und Frauen bis zum Alter von 50 Jahren weiterarbeiten und sich dann die angesparte Summe auszahlen lassen. 77 Es gibt keine Mindestbeitragszeiten und keine Mindest- und Höchstbeträge. Die Auszahlung erfolgt in Form einer einmaligen Abfindungsleistung.78 Der Zinssatz wird jährlich neu festgelegt und beträgt mindestens $3 \% .79 \mathrm{Er}$ lag bisher jeweils weit unter dem üblichen Bankensatz. 80 Die Leistungen sind steuerfrei.81

\section{Probleme des Social Security Fund}

Mit dem Pension Scheme hat das Social Security Scheme gemeinsam, daß die Leistungen zu niedrig sind, um davon leben zu können, weil bereits die Löhne und Gehälter aufgrund der hohen Inflationsrate unzureichend sind. Doch ist diese Inflationsanfälligkeit beim Social Security Fund aufgrund seiner Struktur noch weit stärker ausgeprägt. 82 Insbesondere seit der ersten Olkrise im Jahr 1973 ist die Inflationsrate wesentlich schneller gestiegen, als dies für die Löhne der Fall war. Die durchschnittliche Inflationsrate der Jahre 1986 bis 1989 liegt bei mindestens 30 \%, während den Lohnerhöhungen in diesen Jahren Inflationsannahmen von 15 bis $20 \%$ zugrundeliegen. 83 Die Löhne decken nicht einmal die Ausgaben für die Grundbedürfnisse von Unterkunft, Emährung, Bekleidung etc. ab. Der derzeitige Mindestlohn liegt bei 218 Cedi pro Tag, d.h. bei 6.540 Cedi pro Monat. Nach dem derzeitigen Wechselkurs von etwa 200 entspricht dies 32,50 DM pro Monat. Dies wurde als "grossly inadequate" bezeichnet, da mindestens 500 bis 1.000 Cedi pro Tag und Person benötigt würden, um die Lebenshaltungskosten zu decken. 84

Das zentrale Problem des Social Security Fund besteht darin, daß er nicht, wie echte Rentensysteme, auf dem Sozialversicherungsprinzip aufbaut, wonach das allgemeine Prinzip der Äquivalenz von Beitrag und Leistung durch das Solidaritätsprinzip modifiziert wird. Die darin liegende Risikoverteilung zwischen den Mitgliedern des Fonds wird u.a. durch

76 Nach dem Social Security Act, 1965 hatte das Rentenalter 60 Jahre für Männer und 55 Jahre für Frauen betragen.

77 Ss. 40(1) (a), (b) NRCD 127.

78 Kludze, op. cit., S. 193.

79 Ss. $22,40(2)$ NRCD 127.

80 Kludze, op. cit., S. 189.

81 S. 38 NRCD 127.

82 Vgl. ILO, op. cit., S. 6.

83 West Africa, wöchentliche Zeitschrift, London, 11.-17.6.90, S. 973 f.

84 West Africa, 26.3.-1.4.90, S. 484 f., 514; 11.-17.6.90, S. 996. 
die Festlegung einer Mindestbeitragsdauer und einer Höchst- und Mindestrente erreicht; derartige Festlegungen kennt das Social Security Scheme aber nicht. Insbesondere wo die Arbeitsdauer insgesamt nicht sehr lang war, was wegen des instabilen Arbeitsmarktes häufig der Fall ist, sind die Leistungen aus dem Social Security Fund extrem unzureichend, da keine größeren Beträge angespart werden konnten. Kludze 85 merkt des weiteren an, da $\beta$ der Social Security Fund auch nicht i.S. eines sog. Generationenvertrags angelegt ist, nach dem die Leistungen zumindest teilweise durch die Beiträge der derzeit wirtschaftlich aktiven Bevölkerung erbracht werden.

Ein weiteres Problem liegt darin, daß Leistungen aus dem Social Security Fund ausschließlich als einmalige Abschlagszahlung gewährt werden und dem Rentner somit keine regelmäßigen Leistungen zur Verfügung stehen. Einmalige Zahlungen sind jedoch erfahrungsgemäß zur kontinuierlichen und verläßlichen Alterssicherung nur sehr bedingt geeignet.86 Obwohl einerseits hierdurch Investitionen zur Begründung eines Geschäfts o.ä. erleichtert werden, werden einmalige Abschlagszahlungen andererseits oft innerhalb kürzester Zeit ausgegeben und entfallen dann als längerfristige Alterssicherung. 87 Aufgrund der Inflation reicht heute die Abschlagszahlung nicht mehr für größere Investitionen aus, die ein ausreichendes kontinuierliches Einkommen sichem könnten. 88

Der Social Security and National Insurance Fund (SSNIT) war bisher in seinen Möglichkeiten, effektiv zu wirtschaften, insofem empfindlich beschränkt, als er das aus den Beiträgen eingenommene Treuhandvermögen lediglich in Staatsanleihen (government securities) anlegen durfte, 89 was keine höheren Zinseinnahmen als von $6 \%$ einbrachte, während der allgemeine Bankzins sehr viel höher lag.90 So erklärt sich auch der den Mitgliedem gewährte niedrige Zins auf ihre angesparten Guthaben (s.o.). Andererseits war der Staat Nutznießer dieser Regelung, da ihm hierdurch billige Kredite zur Verfügung standen.

Ein weiteres Problem hängt mit dem bisherigen Rentenalter zusammen (ähnliches gilt für das Pensionsalter nach dem Pensions Scheme). Im Alter von 45, 50 oder 55 Jahren hat ein Arbeitnehmer normalerweise seine finanziellen Verpflichtungen, wie die Ausbildung seiner Kinder, die Unterstützung von Verwandten, den Bau eines Hauses, etc., noch nicht erfüllt bzw. abgeschlossen und ist insoweit noch auf höhere Einkünfte angewiesen als die niedrigen Social Security-Leistungen. Es stellt sich außerdem die Frage, ob ein Entwicklungsland es sich leisten kann, seine qualifizierten Arbeitnehmer in einem Alter in den Ruhestand zu

85 Op. cit., S. 194.

86 Mouton, Pierre: Social Security in Africa. Trends, Problems and Prospects, Geneva, Intemational Labour Office, 1975, S. 119.

87 Kludze, op. cit., S. 193.

88 Asamoah/Nortey, op. cit., S. 37.

89 R. 37(1) SSR.

90 Nach Kludze, op. cit., S. 195,1988 bei $20 \%$. 
schicken, in dem sie einen hohen Grad an Erfahrung und Produktivität zu bieten haben. ${ }^{91}$ In der gegenwärtigen Situation ist es vielfach üblich, daß mit dem Eintritt in den Ruhestand ein eigenes privates Geschäft oder Unternehmen begonnen oder fortgeführt wird, oder daß man in sein Heimatdorf zurückkehrt und sich dort landwirtschaftlich betätigt, um auf diese Weise die unzureichenden Renten aufzubessern. Diese Fortsetzung des Arbeitslebens auf einer anderen Ebene ist fast zur Selbstverständlichkeit geworden, dient aber nicht unbedingt dem wirtschaftlichen Wohl des Landes.92

Ein anderes Problem des Social Security Fund scheint die nicht immer effiziente Verwaltung zu sein. Arbeitgeber, die ihre Beiträge nicht leisten, haben keine ernsten Konsequenzen zu befürchten. Das Fehlen einer administrativen Infrastruktur im Lande, wie z.B. von verläßlichen Personenstandsregistem, Probleme des Postsystems, etc., verursachen ebenfalls Schwierigkeiten.93

Schließlich ist es wichtig, darauf hinzuweisen, daß die große Mehrheit der Bevölkerung, d.h. diejenigen, die in keinem formellen Arbeitsverhältnis stehen und somit die staatliche soziale Alterssicherung jedenfalls bisher nicht oder nur begrenzt in Anspruch nehmen können, im Alter ganz und gar von anderen Einkommensquellen abhängen. Dies betrifft in erster Linie die Bauern, Fischer, Hausangestellten, und die kleinen, im informellen Sektor tätigen Selbständigen oder Angestellten.94 Der Grund hierfür liegt in dem grundsätzlich am formellen Arbeitsverhältnis anknüpfenden Konzept sozialer Sicherheit in den meisten Entwicklungsländern: Staatliche soziale Sicherheit wurde dort zum Privileg der Arbeitnehmer im formellen Sektor 95 und insbesondere der mittleren und höheren Einkommensgruppen. 96

Insgesamt muß der Social Security Fund daher als ein deutlich unzureichendes System sozialer Alterssicherung betrachtet werden. Eine wirkliche Existenzsicherung im Alter wurde durch ihn nicht gewährleistet.97 Erst nach sehr langen Beitragszeiten entsteht ein wesentlicher Leistungsanspruch, was angesichts der instabilen Arbeitsmarktlage häufig ausgeschlossen ist. Die einmalige Abfindungsleistung gewährleistet kein kontinuierliches Einkommen im Alter. Schließlich ist dieses System besonders anfällig für die Auswirkungen der Inflation, die in Ghana, wie in vielen anderen afrikanischen Ländern, extreme Ausmaße angenommen hat.

91 Vgl. Mouton, op. cit., S. 121.

92 Vgl. hierzu People's Daily Graphic, 4.2.88.

$93 \mathrm{Vgl}$. Asamoah/Nortey, op. cit., S. 60.

94 Asamoah/Nortey, op. cit., S. 59.

95 Vgl. Turner, op. cit., S. 23.

96 Zacher, op. cit., S. 32; Asamoah/Nortey, op. cit., S. 60.

97 Mirror 19.3.88. 
Aus diesen Gründen hatte die Internationale Arbeitsorganisation bereits 1960 die Empfehlung ausgesprochen, daß der damals in Ghana einzurichtende Social Security Fund nach fünf Jahren in ein echtes Rentensystem umzuwandeln sei.98 Auch in anderen Ländem wurden Provident Funds lediglich als Ubergangsregelung und Vorstufe zu einem echten Rentensystem betrachtet.99 Lange geschah nichts, um diese Umwandlung tatsächlich durchzuführen. 1001982 wurde jedoch von der ghanaischen Regierung ein Komitee eingesetzt, um das System der sozialen Sicherheit zu revidieren.101 1988 legte der Social Security and National Insurance Trust (SSNIT) einen Vorschlag vor, das bisherige Zwangssparsystem in ein echtes Rentensystem umzuwandeln, das landesweit für private und öffentliche Arbeitnehmer gleichermaßen zur Verfügung stehen soll, soweit letztere nicht von speziellen Pensionssystemen abgesichert sind, und dem auch Selbständige beitreten können.102 Durch die Existenz eines effizienten Rentensystems sollte die Notwendigkeit anderweitiger wirtschaftlicher Absicherung auf privater Basis weitgehend entfallen und Arbeitnehmer sollten dazu motiviert werden, in ihrem eigentlichen Beruf auf höhere Gehälter hinzuarbeiten, statt ihre Energie und Zeit auf die Erzielung von Nebeneinkünften zu richten; dadurch sollte die allgemeine Produktivität erhöht werden.103

\section{Das zukünftige Rentensystem (SSNIT Pension Scheme) 104}

\section{Allgemeines}

Das geplante Rentensystem 105 beruht auf dem Sozialversicherungsprinzip, das vom Prinzip der Risikoverteilung und dem Solidartitätsprinzip charakterisiert wird. Es handelt sich um ein Beitragssystem. Die bisherigen Mitglieder des Social Security Fund werden automatisch Mitglieder des neuen Rentensystems. Wer bereits Leistungen aus dem Social Security

98 Kludze, op. cit., S. 195.

99 Fuchs, op. cit., S. 28.

100 Möglicherweise u.a. deswegen, weil die Regierungen kein Interesse daran hatten, diese Möglichkeit billiger Darlehen aufzugeben, denn die in den Social Security Fund eingezahlten Beiträge stehen dem Staat zu niedrigem Zinssatz bis zum Leistungszeitpunkt zur Verfügung. Die Beiträge zum Social Security Fund wurden daher als eine Art zusätzlicher indirekter Besteuerung der Arbeitnehmer bezeichnet, Kludze, op. cit.

101 Kludze, op. cit., S. 195.

102 People's Daily Graphic 4.2.88.

103 Mirror 19.3.88.

104 Die folgenden Ausführungen beruhen v.a. auf dem vom Public Affairs Department des SSNIT (Social Security and National Insurance Trust) veröffentlichten Informationsblatt "SSNIT Pension Scheme", Accra, o.J.

105 Der Begriff "Pension Scheme" wird hier mit "Rentensystem" wiedergegeben, um deutlich zu machen, daß es sich beim SSNIT Pension Scheme, im Gegensatz zum Public Pension Scheme für Beamte, um ein Beitragssystem für Angestellte handelt. 
Fund erhalten hat, kann diese, zusätzlich eines Zinssatzes von $6 \%$, zurückzahlen und erwirbt dadurch die Möglichkeit, in das SSNIT Pension Scheme einzutreten. Auch die Pensionssysteme der staatlichen und halbstaatlichen Institutionen, die bisher durch das allgemeine Steueraufkommen finanziert werden, sollen in dem SSNIT Pension Scheme aufgehen. Dies gilt jedoch nur für die Angehörigen des sonstigen öffentlichen Dienstes (other public services, s.o.), während die Beamten der allgemeinen Staatsverwaltung (civil service) weiter unter die Pensions Ordinance fallen. Die Angehörigen des Militärs sind nach einer vorliegenden Quelle106 von dem neuen Pension Scheme ausgeschlossen; nach einer anderen Quelle107 können sie nach ihrer Pensionierung noch beginnen, Beiträge zum SSNIT Pension Scheme zu leisten, und nach Erreichen der Mindestbeitragsdauer (was aufgrund des frühen Pensionierungsalters der Militärangehörigen durchaus möglich ist) Renten nach diesem Scheme in Anspruch nehmen.

Der Erfolg des neuen Pension Scheme hängt zum großen Teil von einem effizienten Investitionsprogramm ab. Die Beschränkung auf ausschließliche Anlage in Staatsanleihen etc. wird aufgehoben. Der'SSNIT such daher nach lukrativeren Anlagemöglichkeiten für die eingenommenen Beiträge und hat bereits Anteile verschiedener nichtstaatlicher Unternehmen erworben, um das neue Scheme abzusichern. 108 Seit kurzem konnte, aufgrund bereits gelockerter Vorschriften, durch Anlage in Anteilen ausländischer Unternehmen der Zinsertrag auf bis zu $16,5 \%$ gesteigert werden. 109

\section{Versicherter Personenkreis}

Das neue Rentensystem soll landesweit einheitlich für alle Erwerbstätigen gelten. Während bisher nur Unternehmen mit mindestens fünf Angestellten beitreten konnten und mußten, soll nun eine Pflichtregistrierung aller Unternehmen erfolgen. 110 Insbesondere sind nicht nur Angestellte im formellen Sektor mit permanenten Arbeitsverhältnissen, sondern auch Angestellte und Selbständige im informellen Sektor, wie kleine Handwerker, Händlerinnen, Gelegenheitsarbeiterinnen, Schneiderinnen, etc., berechtigt, Mitglieder zu werden. Wie in den Erläuterungen des SSNIT zutreffend festgestellt wurde, "the concept of old-age and the contingency of death are not the prerogative of those working in the formal sector".

106 Mirror 7.7.90.

107 SSNIT, op. cit.

108 Mirror 7.7.90.

109 Mirror 19.3.88 und 7.7.90.

110 Mirror 7.7.90. 


\section{Beiträge}

Die Beiträge sollen wie bisher unter dem Social Security Decree zu $5 \%$ des Monatseinkommens vom Arbeitnehmer und zu 12,5\% des Einkommens vom Arbeitgeber aufgebracht werden. Selbständige werden als Arbeitgeber und Arbeitnehmer in einer Person angesehen und müssen den Gesamtbeitrag von $17,5 \%$ allein aufbringen. Im Falle von Selbständigen wird das Einkommen, auf dessen Basis der Beitragssatz errechnet wird, von dem Selbständigen selbst festgelegt. Wenn nötig, wird in Fällen von unangemessen hoch erscheinenden Einkünften die Angabe des Selbständigen nachgeprüft, z.B. durch Rücksprache mit den Steuerbehörden, um sicherzustellen, daß auch der für die deklarierten Einkuinfte zutreffende Einkommensteuersatz gezahlt wird. Außer der Einkommens- und damit Beitragshöhe können Selbständige auch die Beitragszeiten selbst festlegen, z.B. im Falle saisonabhängiger Einkommen. An diese Festlegung sind sie dann gebunden.

Die Verwaltungsstruktur des SSNIT soll beibehalten werden.111 Die Beiträge sind bar oder durch Scheck beim nächsten SSNIT-Büro, unter Vorlage einer Beitragsliste, einzuzahlen. Bei Nichterbringen der Beiträge werden Strafzuschläge erhoben, die sich nach bestimmten Prozentsätzen der ausstehenden Beiträge berechnen, um insbesondere im Hinblick auf die zur Finanzierung des Rentensystems vorzunehmenden Investitionen regelmäßige Beitragsleistungen zu erzwingen.

\section{Leistungsvoraussetzungen und Leistungsinhalt}

Die Leistungen nach dem SSNIT Pension Scheme umfassen periodische Geldzahlungen bei Alter, Invalidität und Tod. Sie können neben bestehende betriebliche Systeme treten, die vielfach bei Eintritt in den Ruhestand eine einmalige Zahlung vorsehen (end of service benefit schemes). 112

Voraussetzung für den Anspruch auf die Altersrente soll eine Mindestversicherungs- und damit Beitragszeit von 240 Monaten, d.h. 20 Jahren, sein. Festgelegt wurden eine Mindestrente von $50 \%$ (bei einer Arbeits- und Beitragszeit von 20 Jahren) und eine Höchstrente von $80 \%$ (bei einer Arbeitszeit von 40 Jahren) des Durchschnittsverdienstes der drei besten Verdienstjahre.113 Für jedes über 20 Jahre hinausgehende weitere

$111 \mathrm{Vgl}$. NRCD 127.

112 Vgl. West Africa, 19.-25.3.90, S. 467.

113 So der Entwurf des SSNIT. In der ghanaischen Presse, z.B. im Mirror, 19.3.88, ist mehrfach wohl irrtümlicherweise von den drei letzten Verdienstjahren die Rede. 
Beitragsjahr werden zu den genannten $50 \%$ weitere $1,5 \%$ hinzugerechnet. ${ }^{114}$ Der Grund für die Verwendung der "besten drei (Verdienst-)Jahre" als Bezugsgröße zur Errechnung der Leistungen ist zum einen, um den einmal erreichten relativen Einkommensstandard zu erhalten, und zum anderen um Mißbrauch auszuschließen, der, insbes. im informellen Sektor, bei Verwendung der "letzten drei (Verdienst-)Jahre" leichter möglich wäre.

Das Rentenalter soll für Männer und Frauen gleichermaßen bei 60, auf Antrag bei 55 Jahren liegen; in letzterem Fall werden die Leistungen allerdings gekürzt.115 $25 \%$ der Rente können, statt als regelmäßige Zahlungen, als einmalige Kapitalsumme ausgezahlt werden; in diesem Fall werden die monatlichen Zahlungen entsprechend gekürzt. Die monatliche Rentenauszahlung erfolgt durch eine Bank der Wahl des Rentenberechtigten.

Die Rentenleistungen erfolgen ohne zeitliche Einschränkung bis zum Tod des Rentenberechtigten.

Der Entwurf sieht eine regelmäßige Anpassung der Leistungen an die steigenden Lebenshaltungskosten vor, die sich nach dem gleichen Index wie die Anpassung der Löhne richten soll.

Bei während der aktiven Arbeitszeit eintretender Invalidität werden entweder der zu dem jeweiligen Zeitpunkt dem Betreffenden zustehende Rentensatz geleistet, oder, vorausgesetzt mindestens zwölf Monatsbeiträge wurden während der letzten 36 Monate erbracht, $50 \%$ des letzten Durchschnittseinkommens, je nachdem was höher ist. Die Invalidität ist von einem medizinischen Gremium festzustellen und ihr Andauem in regelmäßigen Zeitabständen zu bestätigen.

Bei Tod des Rentenberechtigten haben unter bestimmten V'oraussetzungen seine von ihm benannten Hinterbliebenen (nominated dependants) einen Anspruch. Folgende Fallvarianten kommen in Betracht: (a) Stirbt ein Rentner nach seinem 72. Lebensjahr, so erfolgen keine Leistungen an seine Hinterbliebenen; (b) stirbt ein Rentner vor seinem 72. Lebensjahr, so wird eine Kapitalabfindung in Höhe der Rentenleistungen, die der Verstorbene bis zu seinem 72. Lebensjahr erhalten hätte, wenn er nicht gestorben wäre, an die Hinterbliebenen geleistet; (c) stirbt ein Erwerbstätiger nach Erreichen der Mindestbeitragsdauer von 20 Jahren, so wird an die Hinterbliebenen eine Kapitalabfindung gezahlt, die dem Betrag entspricht, den er während zwölf Jahren in der Höhe erhalten hätte, auf die er zu seinem

114 Die Leistungssätze betragen daher: nach 20 Jahren: $50 \%$ des Durchschnittseinkornmens der drei besten Verdienstjahre; nach 25 Jahren: 57,5\%; nach 30 Jahren: $65 \%$; nach 35 Jahren: 72,5\%; nach 40 Jahren: $80 \%$.

115 Diese redurierte Rente beträgt: bei 55 Jahren Lebensalter: $60 \%$ der vollen Rente; bei 56 Jahren: 67,5\%; bei 57 Jahren: $75 \%$; bei 58 Jahren: 82,5\%; bei 59 Jahren: $90 \%$. 
Todeszeitpunkt Anspruch hatte; (d) stirbt er vor Erreichen dieser Mindestbeitragsdauer, so wird trotzdem sein bis dahin errichter Rentenanspruch berechnet und der Betrag an die Hinterbliebenen ausgezahlt, den er während zwölf Jahren erhalten hätte.

\section{Schlußbemerkung}

Die Umwandlung des Zwangssparsystems des Social Security Scheme in ein echtes Rentensystem, wie mit dem SSNIT Pension Scheme vorgeschlagen, ist, sollte sie tatsächlich wie geplant erfolgen, ein wichtiger und lange fälliger Schritt zur Verbesserung der staatlichen Alterssicherung.116 Es ist zu hoffen, daß ein entsprechendes Gesetz bald verabschiedet wird und die anerkennenswerten Neuerungen des Entwurfs zielstrebig in die Praxis umgesetzt werden. Der öffentliche Druck hierzu scheint beträchtlich zu sein.

Auch bei optimistischer Einschätzung kann jedoch sicher nicht erwartet werden, daß die Einführung des neuen Rentensystems alle bestehenden und oben angesprochenen Probleme lösen wird, da diese zum großen Teil durch die allgemeine wirtschaftliche Situation bedingt sind.117 Wie Y. Asamoah und D.N.A. Nortey zutreffend schreiben, "given the continued economic constraints the country is likely to experience, expectations about the impact of social security must remain modest".118 Mit H.F. Zacher ist daher davon auszugehen, daß auch das neue SSNIT Pension Scheme auf längere Zeit hin lediglich ein Element einer umfassenderen Strategie sozialer Sicherung sein kann, die Zacher "social security pluralism" nennt.119 Nicht-staatliche Formen sozialer Sicherung bleiben somit, auch wenn sie ihre eigenen, eher zunehmenden Probleme und Unzulänglichkeiten haben, von größter Bedeutung für die Alterssicherung in Ghana. 120

116 Vgl. Kludze, op. cit., S. 208; Asamoah/Nortey, op. cit.

117 Vgl. ILO, op. cit., S. 64.

118 Op. cit., S. 60.

119 Op. cit., S. 32.

120 Leider erlaubt es der zur Verfügung stehende Raum nicht, in diesem Aufsatz näher hierauf einzugehen. Verwiesen sei beispielsweise auf Rwezaura, op. cit.; Kludze, op. cit.; Asamoah/Nortey, op. cit., S. 23, 38, $60 \mathrm{f}$. 


\section{Errata (aus VRÜ 1991, S. 241)}

In VRU 1990, Heft 4, fanden sich zu unserem großen Bedauem Fehler, die wie folgt zu berichtigen sind:

S. $274 \mathrm{ff}$. (Wollny, México y el reto del asilo ...)

S. 374, Zeile 9 - 12:

"también a nivel intemacional México tiene fama de ser uno de los países clásicos de asilo imagen que el país se ha ganado no sólo por sus esfuerzos en pro de los refugiados españoles, sino también, después de los cambios políticos en su propio continente, por la amplia acogida que ha dado a miles de refugiados latinoamericanos en los años setenta."

\section{S. 387, Zeile 11 - 16:}

"Lo que sí es cierto es que en México existen muchos refugiados más que sólo los 40,600 guatemaltecos y los 6,000 de otras nacionalidades que a fines de 1987 recibieron ayuda de ACNUR. Sin embargo, semejante actividad de ACNUR sólo se había producido en Honduras $(40,400$ refugiados asistidos: 15,000 salvadoreños, 25,000 nicaragüenses, 400 guatemaltecos) y Costa Rica (23,100 refugiados asistidos: 3,700 salvadoreños, 19,000 nicaragüenses, 400 refugiados de otras nacionalidades)."

\section{S. 392, Zeile 5 - 9:}

"Los problemas de seguridad que cré la problemática de los refugiados, influyeron notoriamente en la política exterior mexicana: mientras con José López Portillo se mostró todavía una simpatía abierta hacia las revoluciones sociales en Centroamérica, a partir del gobiemo de Miguel de la Madrid, México adoptó una posición mucho más moderada, apoyando especialmente la búsqueda de soluciones negociadas."

S. 393, Zeile 13 - 17:

"El art. 33 de la Constitución Política de 1917, aplicable tanto a los asilados como a todos los otros extranjeros, dice al respecto: '... (Los extranjeros) tienen derecho a las garantías que otorga el capítulo I, titulo primero, de la presente Constitución; pero el Ejecutivo de la Unión tendrá la facultad exclusiva de hacer abandonar el territorio nacional, inmediatamente y sin necesidad de jucio previo, a todo extranjero cuya permanencia juzgue inconveniente. (...)' Según ..."

S. 397 ff. (Wanitzek, Staatliche Systeme sozialer Sicherheit in Afrika ...)

S. 402, letzte Zeile: Pensionsberechtigten

S. 407, Fußnote 77: Ss. 40 (1) (a), 45 (a), (b) NRCD 127 


\title{
ABSTRACTS
}

\section{Mexico and the Challenge of Asylum: A Vision from Abroad}

\author{
By Hans Wollny
}

Surveying Mexico's asylum policy throughout the last fifty years, the article examines the country's varying attitudes toward refugees from Spain and other European countries before and during World War II, from Latin America's Southem Cone in the 70s, and from Central America since the beginning of the 80s. Notwithstanding the country's contributions to the solution of urgent refugee situations which laid the foundations of Mexico's traditional reputation as one of the classical asylum countries, the article shows that Mexican asylum policy was never based exclusively on purely humanitarian purposes, but has always been heavily influenced by aspects of political affinity, of employment and population policies and of national development strategies. Till now, applicable asylum regulations supported this tendency by leaving the decision on refugee questions exclusively within the domain of the executive. Thus, legal provisions served rather as a shield against unwanted refugee groups than as a safe ground for the refugee himself.

Support of the Elderly by the State: The Pensions and Social Security Schemes of Ghana

\section{By Ulrike Wanitzek}

In the days when subsistence economy was a characteristic feature of life in Africa, the protection and support of the elderly was the concem of the extended family. Since the introduction of the money economy, industrialisation and urbanisation, it has become increasingly difficult for the family alone to cope with these obligations. In most countries of Africa, including Ghana, the state has, therefore, taken measures to complement the efforts of the traditional social system.

This paper examines what the state has been able to do in this field. The first measure was the passing of the Pensions Ordinance in 1950 which provided for a non-contributory system of pensions for civil servants only. Later, in 1965, the Social Security Act, as amended later by the Social Security Decree of 1972 , was put in place to provide a system 
of the payment of one lump sum on the retirement of other public servants and private employees. Unlike the Pension Scheme, the Social Security Scheme is a contributory system under which both employers and employees contribute to a Fund; and it is a mere compulsory saving system. Members of the Scheme become entitled to receive sufficient benefits only after they made contributions over a long period of years. However, even then the value of the amount paid on retirement is considerably diminished because of inflation. Hence the dissatisfaction with the existing system of social security.

In view of this, the labour force urged the Govemment to convert the social security system into a pension scheme available for everybody. A new Pension Scheme was then drafted and suggested by the Social Security and National Insurance Trust to the Government. Everybody, including selfemployed persons, can become a member and contribute to the Fund. After a minimum period of 20 years a person qualifies for pension benefits. The current periodical payments of pension benefits are reviewed following changes in the general level of eaming in order to take into account the effects of inflation. Since the success of the Scheme depends to a great extent on the adoption of a sound investment programme, the existing restrictions on investments are removed.

It is expected that the new Pension Scheme will become law soon. If this will be the case, one has to wait and see how successful it will be in giving more lasting security to the elderly than was the case under the existing Social Security Scheme.

\title{
Some Comments on Recent Developments in the Indonesian Marriage Law with Particular Respect to the Rights of Women
}

\author{
By S. Pompe and J. M. Otto
}

The 1974 Marriage Law of Indonesia has been generally acclaimed as an innovative piece of legislation. In this article, however, it is argued that notably in the field of rights of women in Muslim marriages its implementation is not without problems. This article establishes that the structure of the law in these matters is to leave the rights accorded to man in Islam intact, but encumbering their use by means of procedural obstacles. In theory the result ist to give the women in Muslim marriage a better protection in law. It is then suggested that on the basis of the data presently available, the implementation ot the law is flawed in three respects. First, there appears to be a general tendency amongst the religious vourts competent in these matters to improperly apply the law. Second, the religious courts do not appear to heed Supreme Court jurisprudence. Third, the religious courts are understaffed and overworked. These factors threaten the protection accorded to women in Muslim marriage by the Marriage Law. 